\title{
Implementation of a waveform recovery algorithm on FPGAs using a zonal method (Hudgin)
}

\author{
J.J. Díaz ${ }^{1, a}$, A. Dávila-González², L.F. Rodríguez-Ramos ${ }^{1}$, J.M. Rodríguez-Ramos ${ }^{2}$, Y. Martín ${ }^{1}$, and \\ J. Piqueras ${ }^{1}$ \\ 1 Instituto de Astrofísica de Canarias, Santa Cruz de Tenerife 38205, Spain \\ 2 Universidad de La Laguna, Tenerife, Spain
}

\begin{abstract}
The advent of Extremely Large Telescopes impose the existence of wavefront correctors to achieve diffraction limited images. The light collection area is more evidently affected by atmospheric turbulences and image distortion is more relevant than in smaller telescopes. AO system where mostly used to improve the performances while now turn to be a real need. Though the existence of a wavefront reconstructor is mandatory for the next generation of telescopes, the requirements imposed for such large apertures are to be examined and the new challenges must be faced.
\end{abstract}

\section{Introduction}

Changes in the diffraction index of the media, due to variations in parameters affecting the atmosphere, produce wave front distortions. These aberrations affect the spatial resolution of the objects and disperse the photons in a bigger area on the detector making it less effective in the detection of faint object. Today, and next generation telescope developments, claim for the detection of faint objects while obtaining the best spatial resolution possible. If with a $10 \mathrm{~m}$ telescope, located in an observatory with optimum observing conditions, a 0.4 arcsec structure can be resolved, when this resolution is improved down to 0.04 arcsec, the resulting system performance is similar to what could be obtained in a $100 \mathrm{~m}$ telescope under the same atmospheric conditions with no wavefront correction. This gives an idea of the limitations introduced by the atmosphere and the importance of AO systems in the next generation of telescopes where diameters from 30 to 100 meters are the goal. Today most observatories provide AO facilities to allow a better spatial resolution as an additional feature. For next generation of telescopes the huge diameters make it mandatory.

\section{AO SYSTEM}

\subsection{Functional Description}

The objective of an AO system that we consider here is the correction of the wavefront that reaches the telescope with aberrations due to distortions introduced by the atmosphere along the light path. The AO system requires three functions: To detect the wavefront, determine what waveform should have reached the telescope in absence of a distorting media such as the atmosphere, and generate all the required steps to produce a correction to compensate for the aberrations. This divides the AO system in three main subsystems:

- The waveform sensor: Detects the light entering the telescope

- The waveform detection: Using the information obtained from the waveform sensor determines the local variations of the waveform

- The waveform recovery algorithm: Using the information provided by the local variations of the incoming waveform recovers the information of the waveform that should have reached the telescope in absence of distortion.

\footnotetext{
a e-mail: jdg@iac.es
} the original work is properly cited. 


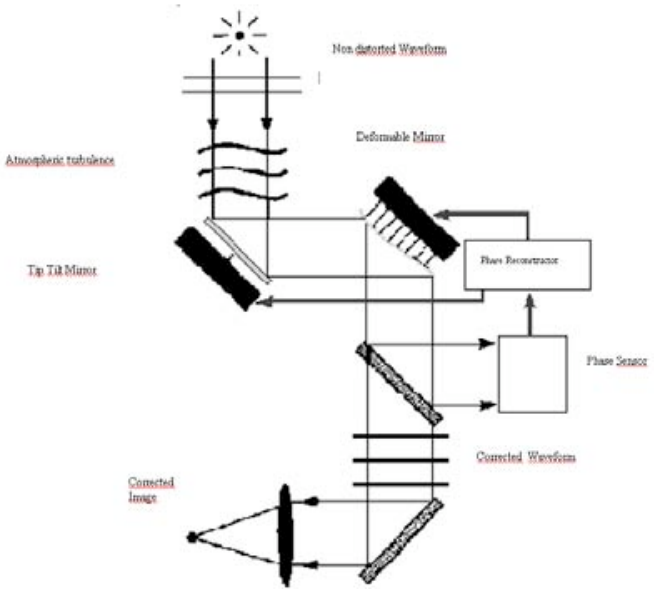

Fig. 1. AO system components

- The calculation of the actuations required by the compensating component: Once the waveform information is known then the compensation parameters that need to be applied to the compensator are derived.

- An active component: Usually a deformable mirror that may adapt to the required shape to compensate from the aberration.

\subsection{Hardware}

A Shark-Harmann sensor is a well known waveform sensor that uses an array of microlenses in front of a detector to project subapertures of the waveform on it. The image of every single subaperture follow into a section of the detector and, in the case of absence of distortion, a single light point would be projected into the corresponding section of the detector. The effect of the aberration results in a displacement of such projection producing dx-dy displacements values for each subaperture.

The number of microlenses that compose the array determines the spatial sampling of the waveform. Every subaperture is projected into a section of the detector where the displacement has to be computed. The number of pixels corresponding to a subaperture determines the accuracy in resolving the displacement of the subaperture projection dx-dy.

For Extremely Large Telescopes (ELTs), with collecting apertures from 30 to 100 meters, the size of the focal plane is bigger than for present telescopes. Even if the spatial sampling resolution is to be maintained, the total number of subapertures will increase with a power of two factor. It is thus advisable to assume that the number of apertures required to obtain a reasonable spatial sampling is in the order of $32 * 32$, which means that a $32 * 32$ or larger microlenses array will be used.

Also, to allow a better resolution in the gradient of the waveform in a certain subaperture, as a result of the atmospheric distortion and indicated by the displacement of the projection of every projection on the detector, the number of pixels per subaperture should be increased. To be on a safe side a number of $32 * 32$ pixels per subaperture is considered.

After the detection of photons using the Shack-Hartmann sensor, the gradient of the waveform for each subaperture needs to be computed. Two matrixes DX and DY, with the dx and dy values corresponding to the deviations of the centroid from the nominal position for every subaperture, are obtained. Different methods have been used:

- Centroiding or Gravity method: this method uses the well known gravity or weight method where every pixel contributes with a factor corresponding to the total signal received multiplied by a factor representing the distance in $\mathrm{x}$ or $\mathrm{y}$ to the nominal position in the non distorted waveform.

- Image Correlation: image correlation techniques are applied to each subaperture to compare consecutive images and to determine the DX and DY matrixes. 
J.J. Díaz et al.: Waveform recovery algorithm on FPGAs using a zonal method

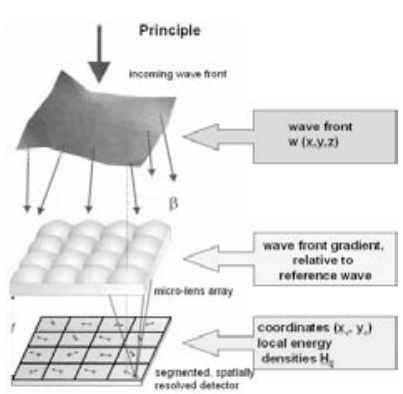

Fig. 2. Shack-Hartmann Sensor

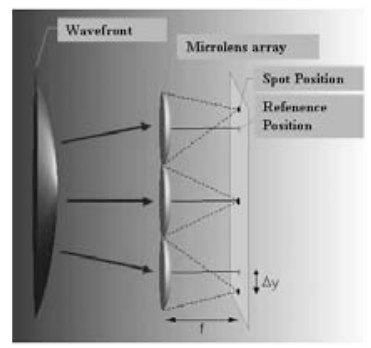

Fig. 3. Projection of subapertures onto the detector for a Shack-Hartmann sensor.

- Waveform Recovery: Once the information of the local variations, gradients, of the waveform for every subaperture is known, then the next step is to obtain the value of the waveform starting from the information contained in the DX and DY matrixes. This method is the objective of this work and will be detailed.

- Transformations and Deformable Mirror actuation: once the waveform received is known, then the correction that needs to be applied to obtain a non distorted waveform has to be calculated and the physical signals to be applied to the correcting element, normally a deformable mirror, generated. This is the last process in the sequence that has to be repeated with a time response fast enough to produce the correction before the distortion of the incoming waveform changes significantly.

\section{THE HUDGIN METHOD}

Hudgin provides an iterative mathematical method that allows the reconstruction of a bidimensional function from the information contained in the local variations in both directions. The algorithm is represented by the formula:

$$
\phi^{(M)}=1 / 4\left(\phi_{1}^{(M-1)}+\phi_{2}^{(M-1)}+\phi_{3}^{(M-1)}+\phi_{4}^{(M-1)}+\Delta \phi_{1}+\Delta \phi_{2}+\Delta \phi_{3}+\Delta \phi_{4}\right)
$$

where $\mathrm{M}$ represents the algorithm iteration index, the phase values of neighboring subapertures are represented by $\phi_{1}, \phi_{2}, \phi_{3}, \phi_{4}$ and the gradients are represented by $\Delta \phi_{1}, \Delta \phi_{2}, \Delta \phi_{3}, \Delta \phi_{4}$. The Hudgin method, as is based on local variations of the function, does not allow the recovery of the continuum value of the function. This is not necessary for our purpose as our need is to correct for the distortions.

Some numerical simulations have been performed to understand the capabilities of this method and the feasibility to implement it in FPGAs where the floating point arithmetic is not efficient.

The accuracy of the final result depends on the number of iterations performed. This directly affects the time required to obtain a result that matches, with reasonable error, to the actual waveform reaching the sensor. The actual number of iteration cycles may depend on the characteristics of the waveform function. It has been found that, with little difference, for Gaussian and Kolmogorov like waveforms iterations in the order of 500 give results with error results below $10^{-3}$ being the error in the order of $10^{-2}$ for about 200 iterations. More than 500 iterations will not produce significant error reduction and thus this figure can be considered as the required number of iterations for a complete recovery of the incoming waveform.

The actual application will deal with atmospheric like distorted waveforms. To validate the method it is required to know the initial waveform to compare it with the result obtained after running the method. Then it is necessary to synthesize atmospheric like waveforms. Assuming that Kolmogorov statistics represents a valid model we used waveforms using this statistics. The result was that the method works well for this type of waveforms. 


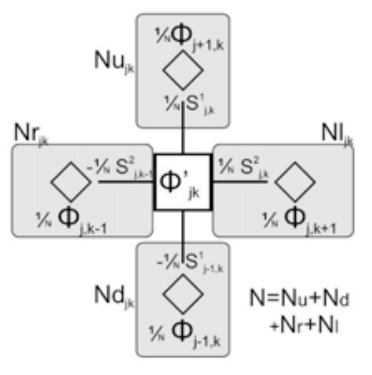

Fig. 4. The Hudgin formula

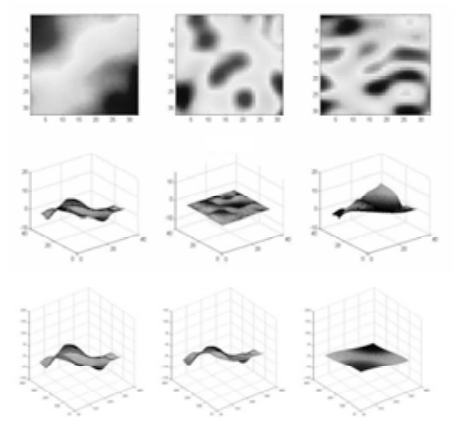

Fig. 5. Waveform reconstruction from an image generated following the Kolmogorof statistics. From Top left to bottom right. Initial waveform, DX-DY matrices and comparison of original and recovered function with error for non border correction (middle) and with border correction (bottom)

\section{Algorithm implementation in FPGAs: feasibility}

Once the validity of the algorithm to recover waveforms as those likely to be produced by the atmosphere has been proved, the next step is to provide a physical implementation of the algorithm into a HW able to perform the required calculations in the time frame imposed by the application. As mentioned before, the total time available to correct from waveform distortion, from the image acquisition till compensation, has to be less than $10 \mathrm{~ms}$. It is then advisable to make this computation, and all the computations and actions required to compensate the aberrations, in the minimum time possible. This work pretends to produce dedicated HW to make this calculations at the fastest speed possible. The circuits will be implemented in FPGA. The HW inside the FPGA is rather efficient if calculations are made in fixed point arithmetic. First it has to be demonstrated that the results, obtained with this arithmetic follows the results in the previous simulations with floating point arithmetic. A couple of assumptions have been made to provide relevant results:

- Number of subapertures:We assume that the maximum number of subapertures is $256 * 256$. This parameter determines the maximum spatial information of the waveform. The spatial resolution.

- Number of pixels per subaperture: We assume that the maximum number of pixels corresponding to the area of the detector associated to each subaperture is $256 * 256$. This determines the maximum resolution in the calculations of the $\mathrm{dx}$ and dy values.

The results obtained using Fixed and Floating point arithmetic have been compared and the error found is in the order of $10^{-3}$. This is in the order of magnitude of the error in the waveform recovered for floating point arithmetic and thus it can be concluded that the use of fixed point arithmetic will not pose any significant error. The HW implementation on FPGA of the circuits to perform the Hudgin algorithm is therefore feasible.

The circuit architecture has been design to facilitate parallelism. To calculate the new phase matrix there will be as many circuits as files of subapertures operating in parallel. All the phases of a particular column of subapertures is calculated in parallel.

The process flow is controlled using a state machine that performs the same operations in every iteration cycle. The steps followed for every calculation consist of:

- Read the dx, dy and phase values: Required for the calculations of a particular subaperture. As the state machine is based on a column index step, a line phase calculator requires 1 phase value, $2 \mathrm{dx}$ and 2 dy values. This means that the values of 3 consecutive columns are required to obtain the phase corresponding to the aperture under consideration. The result of the first column of phases is calculated after the data from the 2 first columns, plus the assumed values for column -1 , are available. 

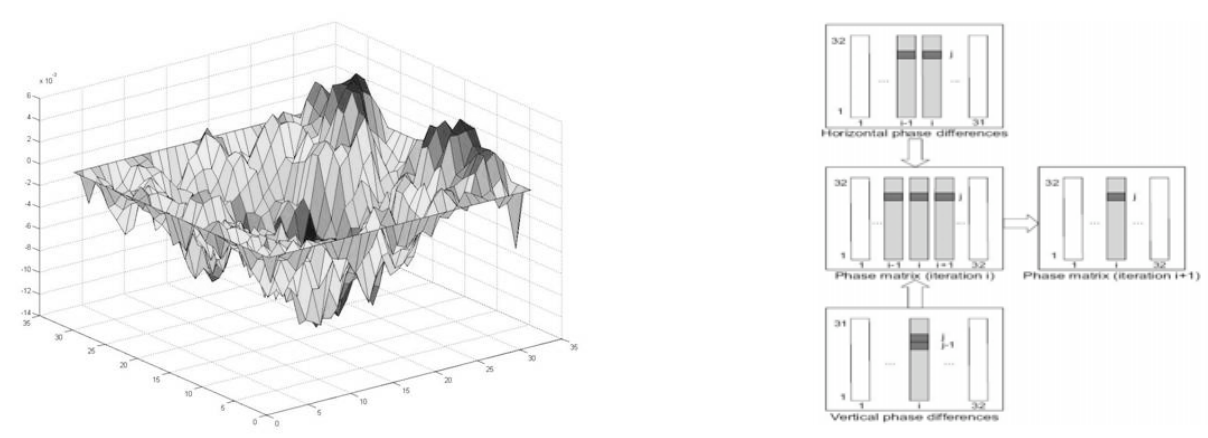

Fig. 6. Differences between Floating point and fixed Fig. 7. Memory architecture with DX-DY and Phase valpoint arithmetic simulations. ues.

- Operate the results: Once the $\mathrm{dx}$, dy and phase values are available then the operations can be performed. There are a couple of singularities:

- The subapertures in the borders do not have neighbours and the missing values for the phase are considered to have the same value as the phase to of the point under consideration during the previous iteration.

- There is no chance to make any operation till the values of the 2 first columns are received. Once the first 2 columns are read then the phase of the first singular column is calculated assuming that there is a column 0 whose phase values equals the phase values of column 1.

- Once this singular section has been covered then a new column of phases will be updated for every new column of subapertures that is read.

- To update the phase matrix: Once the new value of the phase has been calculated, before reading a new column of phases, the new values of the column are updated in the phase memory.

To provide the results and make an estimation of the benefits given by this hardware approach the circuit has been implemented into a VIRTEX 5 FPGA from Xilinx. It is considered for $32 * 32$ subapertures and uses 16 bit resolution.

\section{Accuracy results and time performance}

The testing system was designed to allow introducing the same DX, DY matrixes as used in the mathematical computations. The phase matrix was recovered and stored in a file after the simulation of a circuit run. This resulting matrix was compared to the results obtained numerically. The same result was obtained from both methods for equal number of iterations and different input waveforms.

A key parameter, that indicated the need of a $\mathrm{HW}$ implementation was the time required to run the algorithm. The result, obtained implementing the circuit in this particular FPGA but with no optimization to accommodate the circuits to the particular FPGA resources, for:

- With a $100 \mathrm{MHz}$ master clock

- A full run with 10 iterations

- For $32 * 32$ sub-apertures and 16 bit resolution

The total time required is $45 \mu \mathrm{sec}$ which means roughly $4: 5 \mu \mathrm{sec}$ per iteration. If the error of the waveform recovered by the algorithm is acceptable after 200 iterations, then $0,9 \mathrm{~ms}$ are required to obtain the initial waveform. This implementation makes it an ideal choice for AO systems such as those required by ELTs, where the number of phase sub-apertures $128 * 128$ or even $256 * 256$ is considered.

- The time performance of this circuit is not a limitation factor in the AO system

- A proper reception sequence of the local variations of the wavefront allows processing in parallel while still receiving the remaining information.

- To allow a convenient sequence of local variation information the corresponding circuit and Phase sensor architecture need to be considered from the design phase. 


\begin{tabular}{l|l|l|l}
\hline $\begin{array}{l}\text { Virtex5 } \\
\text { xc5vlx30-3ff324 } \\
\text { Flip Flops }\end{array}$ & Used & Available & $\%$ Used \\
\hline LUTs & 4700 & 51840 & $9 \%$ \\
\hline Block RAM & 9357 & 51840 & $18 \%$ \\
\hline Slices & 48 & 96 & $50 \%$ \\
\hline Memory (KB) & 2787 & 12960 & $21 \%$ \\
\hline
\end{tabular}

Fig. 8. Memory architecture with DX-DY and Phase values.

\section{Conclusions}

The fastest implementation known for this algorithm is based on GNUs and the performance of this implementation on FPGAs is about 2 orders of magnitude faster.

The optimization effort to improve time performance and resource consumption has been limited and there is still margin to improve the results but with the present results it can be concluded that using the proper HW architecture of the AO system components a noticeable improvement in time performance could be obtained. Some of these key factors are mentioned.

It is advisable to run the algorithms in parallel and apply the corrections to different sections of the waveform as soon as they are available. The waveform variations take place along time and the fastest the correction the better the result.

Referring only to the detector needs in terms of speed there is nothing new in this aspect. It is relevant that the processing of the DX-DY matrices can only been calculated once all the pixels corresponding to the subaperture are available. This indicates that the ideal detector architecture would provide an output channel per aperture and all this channels, al least those channels corresponding to subapertures in the same column of subapertures, are read and delivered with no delay. As this may imply too many outputs, the configurations should be done in such a way that the pixels corresponding to a same column are read at the same time with no dead times.

If the Hudgin algorithm is to be used then the subsystem used to calculate the difference matrixes should deliver the DX and DY values column after column to facilitate the process as planned. Every new column will provide enough information to start running the algorithm to recover the phase of every aperture in the column.

The time performance of this circuit is not a limitation factor in the AO system.

\section{Acknowledgments}

This work has been partially funded by the Spanish Programa Nacional I+D+i (Project DPI 200607906) of the Ministerio de Educación y Ciencia, and also by the European Regional Development Fund (ERDF).

\section{References}

1. J.M. Hernández, Atmospheric wavefront phase recovery using specialized hardware: GPUs and FPGAs, SPIE (2005)

2. J.M.R. Ramos, Detección de frente de onda. Aplicación a técnicas de alta resolución espacial y alineamiento de superficies ópticas segmentadas (2005)

3. Y. Martín, Fixed-point vs Floating-point arithmetic comparison for adaptative optics real time control computation 